\title{
Dense core evolutions induced by shock triggering and turbulent dissipation
}

\author{
Kengo Tachihara ${ }^{1}$, A. Hayashi ${ }^{2}$, T. Onishi ${ }^{2}$, A. Mizuno ${ }^{3}$ \\ and Y. Fukui ${ }^{2}$ \\ ${ }^{1}$ Graduate School of Science and Technology, Kobe University, 1-1 Rokko-dai, Nada-ku, Kobe, \\ 657-8501, Japan \\ email: tatihara@kobe-u.ac.jp \\ ${ }^{2}$ Department of Astrophysics, Nagoya University, Furo-cho, Chikusa-ku, Nagoya, 464-8602, \\ Japan \\ ${ }^{3}$ Solar-Terrestrial Environment Laboratory, Nagoya University, Furo-cho, Chikusa-ku, Nagoya, \\ 464-8602, Japan
}

Keywords. stars: formation, ISM: clouds, ISM: evolution, ISM: molecules, ISM: structure, radio lines: ISM

\section{Introduction}

External shock triggering and internal turbulence play major role for the condensation of the ISM and star formation. Some evidences of shock triggering by non-isotropic compression are seen in the cloud morphologies and associated active cluster formation such as the $\rho$ Oph and Cha I clouds. Surveys for $\mathrm{C}^{18} \mathrm{O}$ dense cores have shown that internal turbulence dominates the core dynamics and regulates star formation activity (Tachihara et al. 2002).

\section{Observations and results}

Based on the precedent $\mathrm{C}^{18} \mathrm{O}$ core surveys by the NANTEN radio telescope (Tachihara et al. 2002 and the references therein), the Taurus, Ophiuchus North, Lupus, and Chamaeleon clouds were surveyed for denser and more compact cores in $\mathrm{H}^{13} \mathrm{CO}^{+}(J=1-0)$ by the $45 \mathrm{~m}$ telescope at the Nobeyama Radio Observatory and the SEST $15 \mathrm{~m}$ telescope at La Silla. The results obtained in Taurus were published by Onishi et al. (2002). For a comparison, $\mathrm{H}^{13} \mathrm{CO}^{+}$survey with the $45 \mathrm{~m}$ telescope in the $\rho$ Oph cloud by Umemoto et al. (2002) are compiled.

In general, one $\mathrm{C}^{18} \mathrm{O}$ core (typical density is $\sim 10^{4} \mathrm{~cm}^{-3}$ ) fragments into a few $\mathrm{H}^{13} \mathrm{CO}^{+}$cores $\left(\sim 10^{5} \mathrm{~cm}^{-3}\right)$ in isolated star-forming regions (SFRs), in contrast to the typical triggered clusterforming region of the $\rho$ Oph cloud, which consists of $57 \mathrm{H}^{13} \mathrm{CO}^{+}$cores. The statistics show a remarkable trend that more evolved $\mathrm{C}^{18} \mathrm{O}$ cores associated with $\mathrm{H}^{13} \mathrm{CO}^{+}$cores and young stars have larger masses and smaller line widths than those without $\mathrm{H}^{13} \mathrm{CO}^{+}$cores. This suggests that the turbulent decay is required for the dynamical relaxation of the $\mathrm{C}^{18} \mathrm{O}$ cores to gain more mass and then contract to form denser $\mathrm{H}^{13} \mathrm{CO}^{+}$cores spontaneously. On the other hand, no clear trend is seen in the physical properties between star-forming and prestellar $\mathrm{H}^{13} \mathrm{CO}^{+}$cores. Among the nearby SFRs, the $\mathrm{H}^{13} \mathrm{CO}^{+}$cores in Taurus are larger in number and mass than in other SFRs, while the $\rho$ Oph and Cha I clouds have larger mass fractions of the total $\mathrm{C}^{18} \mathrm{O}$ cores to the parental ${ }^{13} \mathrm{CO}$ clouds. We suggest that the external shock compresses the low-density part of the clouds and the internal turbulent decay leads the dense core condensations.

\section{References}

Onishi, T., Mizuno, A., Kawamura, A., Tachihara, K., \& Fukui, Y. 2002, ApJ 575, 950

Tachihara, K., Onishi, T., Mizuno, A., \& Fukui, Y. 2002, A $8 A$ 385, 909

Umemoto, T., Kamazaki, T., et al. 2002, in: S. Ikeuchi, J. Hearnshaw \& T. Hanawa (eds), 8th IAU Asia-Pacific regional meeting (Ast.Soc.Japan), Vol. II, p.229 